\title{
EFICIENCIA FOTOQUÍMICA MÁXIMA E ÍNDICE DE POTENCIAL FOTOSINTÉTICO EN PLANTAS DE MELÓN (Cucumis melo) TRATADAS CON BAJAS TEMPERATURAS
}

\author{
ROdRIGUEZ TORRESSI, A. O.1; YONNY, M.2; \\ Nazareno, M. ${ }^{2}$; Galmarini, C. R. ${ }^{3} \&$ Bouzo, C.A. ${ }^{4}$
}

\begin{abstract}
RESUMEN
La fluorescencia de la clorofila se utiliza para determinar la eficiencia fotoquímica de las plantas ante diferentes condiciones ambientales. Existen índices como Fv/Fm y PI abs que son indicadores indirectos del rendimiento cuántico del fotosistema II (PSII). El objetivo de este trabajo fue determinar la eficiencia fotoquímica máxima del PSII y el potencial fotosintético en plantas sometidas a bajas temperaturas. La experiencia se llevó a cabo en la EEA, Santiago del Estero y en la Facultad de Agronomía y Agroindustria, UNSE. Los tratamientos consistieron en plantas de melón (cv. Sweet Ball) sin estímulo de frío (testigo) y plantas con estímulo de frío durante la noche con rangos térmi$\cos$ de $0^{\circ} \mathrm{C}$ a $10^{\circ} \mathrm{C}$ y de $-3^{\circ} \mathrm{C}$ a $0^{\circ} \mathrm{C}$. Se evaluó la eficiencia fotoquímica máxima ( $\left.\mathrm{Fv} / \mathrm{Fm}\right)$, índice de potencial fotosintético (PI abs) y concentración de malondialdehido (MDA) en hoja. En las plantas estimuladas con frío se obtuvo menor Fv/Fm, PI abs e incrementos en la concentración de MDA. Palabras claves: Cucumis melo sp., fluorescencia, malondialdehido.
\end{abstract}

\section{SUMMARY}

Maximum efficiency phothochemistry and potential photosynthetic index in melon plants (Cucumis melo) treated with low temperaturas.

Measurements of the chlorophyll fluorescence is used to examine the photochemical efficiency of plants a wide range of environmental conditions. The quantum yield of non-cyclic electron transport is directly proportional to the efficiency of excitation of the reaction centers of Photosystem II (PS II) and can be determined by indexes such as Fv / Fm and PI abs. The aim of this study was

1.- EEA-INTA Santiago del Estero. Producción vegetal, sección hortícola. Jujuy No 850. (4200) Santiago del Estero. Email: rodriguez.ariel@inta.gob.ar.

2.- Universidad Nacional de Santiago del Estero, Laboratorio de antioxidantes y procesos oxidativos.

3.- INTA EEA La Consulta - CONICET.

4.- Facultad de Ciencias Agrarias, Universidad Nacional del Litoral.

Manuscrito recibidoe el 26 de mayo de 2014 y aceptado para su publicación el 4 de diciembre de 2014. 


\section{A. O. Rodríguez Torressi et al.}

to determine the maximum photochemical efficiency of PSII and photosynthetic potential in plants treated with low temperatures. The experiment was conducted at the Estación Experimental Agro pecuaria (EEA) INTA y la Facultad de Agronomía y Agroindustria de la Universidad Nacional de Santiago del Estero (UNSE). Treatments consisted of melon plants (cv. Sweet Ball) without (control) and with low night temperatures between $10^{\circ} \mathrm{C}$ and $0^{\circ} \mathrm{C}$ to $-3^{\circ} \mathrm{C}$ to $0^{\circ} \mathrm{C}$. Photochemical efficiency (Fv / Fm), photosynthetic potential index (PI abs) and in turn MDA concentration increased. Key words: Cucumis melo sp., flourescence, malondialdehyde (MDA).

\section{INTRODUCCIÓN}

La energía lumínica captada por las hojas de las plantas sigue tres procesos, que se generan de forma simultánea: el fotoquímico de la fotosíntesis, la disipación como calor y la re-emisión como luz de baja energía o fluorescencia (Moreno et al., 2008).

Estos tres procesos ocurren, de tal manera que el incremento en la eficiencia de uno de ellos, resulta en la disminución de los otros dos. Por este motivo, al medir el rendimiento de la fluorescencia de la clorofila se puede obtener información acerca de los cambios en la eficiencia fotoquímica y de la disipación de calor (Maxwell \& Johnson, 2000). Este fenómeno puede ser medido mediante un fluorímetro, el que permite discriminar entre un nivel mínimo de fluorescencia (Fo) y un nivel máximo (Fm), valor éste que representa la anulación del proceso fotoquímico.

Ante condiciones adversas debida a estrés, se modifican las proporciones del reparto de energía entre estos procesos incrementándose la fluorescencia (Moreno et al., 2008). La diferencia entre los niveles anteriores de Fo y Fm, determina la fluorescencia variable (Fv). Considerando estos parámetros entre sí, la relación entre Fv/Fm permite establecer situaciones de plantas no estresadas cuando su valor se encuentra entre 0,75 y 0,85 (Bjorman \& Demmig, 1987). Esta relación y el índice de potencial fotosintético (PI abs) son por lo tanto indicadores indirectos del rendimiento cuántico del fotosistema II (PSII).

Por otra parte, las condiciones de estrés incrementan la producción de especies reactivas de oxígeno (EROs) (Gulen et al., 2008). Estas moléculas reaccionan con los lípidos de las membranas a través de un proceso conocido como peroxidación lipídica. El grado de avance de esta reacción puede determinarse a partir de la cuantificación de los productos generados por ella, siendo uno de los más estudiados el malondialdehido (MDA) (Esterbauer \& Cheeseman, 1990).

Como hipótesis de trabajo se estableció que en melón, las bajas temperaturas afectan la cadena transportadora de electrones, aumentando la fluorescencia. La baja temperatura, produce daños reversibles e irreversibles, incrementando la concentración de malondialdehido (MDA).

El objetivo de este trabajo fue determinar la eficiencia fotoquímica máxima del PSII y el potencial fotosintético en plantas sometidas a bajas temperaturas.

90 | Revista FAVE - Ciencias Agrarias 13 (1 - 2) 2014 